\title{
Further Study on Dynamics for a Fractional-Order Competitor-Competitor-Mutualist Lotka-Volterra System
}

\author{
Bingnan Tang (iD) \\ Business School, Jiangsu University of Technology, Changzhou 213001, China \\ Correspondence should be addressed to Bingnan Tang; regales1988@sina.com
}

Received 11 June 2020; Accepted 28 January 2021; Published 22 February 2021

Academic Editor: Karthikeyan Rajagopal

Copyright (c) 2021 Bingnan Tang. This is an open access article distributed under the Creative Commons Attribution License, which permits unrestricted use, distribution, and reproduction in any medium, provided the original work is properly cited.

On the basis of the previous publications, a new fractional-order prey-predator model is set up. Firstly, we discuss the existence, uniqueness, and nonnegativity for the involved fractional-order prey-predator model. Secondly, by analyzing the characteristic equation of the considered fractional-order Lotka-Volterra model and regarding the delay as bifurcation variable, we set up a new sufficient criterion to guarantee the stability behavior and the appearance of Hopf bifurcation for the addressed fractional-order Lotka-Volterra system. Thirdly, we perform the computer simulations with Matlab software to substantiate the rationalisation of the analysis conclusions. The obtained results play an important role in maintaining the balance of population in natural world.

\section{Introduction}

For a long time, the dynamic characteristics of interaction between predator population and prey population has been a central issue in ecology and biomathematics due to its general appearance and potential importance [1]. General speaking, the interaction between predator population and prey population includes four cases: competition, predation, mutualism, and parasitism [2]. During the past few decades, a great deal of valuable research fruits on dynamical behavior of the above four-type predator-prey models has been covered. For example, Alidousti and Ghafari [3] investigated the Hopf bifurcation and limit cycle of the fractional-order predator-prey model; Sasmal and Takeuchi [4] studied the stability behavior of all equilibria, bifurcation nature, global features, and multistability of a predator-prey model; Ryu and Ko [5] discussed the asymptotic peculiarity for positive solutions for a prey-predator model; Guo et al. [6] proved the appearance of traveling waves in a prey-predator system. Zhang et al. [7] revealed the effect of the fear factor on the periodic solution of a prey-predator model. As for concrete works, we refer the readers to [8-25].

In real life, any biological or environmental coefficients will change with time. So, the parameters in predator-prey models are usually not fixed constants. They are often functions with respect to time. In particular, the influence of a periodically varying environment on the dynamics of predator-prey models plays a vital role in maintaining population balance. Furthermore, the capture of the prey from the predator throughout its past time has an important effect on the present birth rate of the predator $[26,27]$. Thus, it is of importance to establish the various type predatorprey models with periodic coefficients. Based on this idea, in 2010, Lv et al. [27] established the predator-prey model involving periodic coefficients as follows: 


$$
\left\{\begin{array}{l}
\dot{u}_{1}(t)=u_{1}(t)\left[\gamma_{1}(t)-\alpha_{11}(t) u_{1}\left(t-\sigma_{11}(t)\right)-\alpha_{12}(t) u_{2}\left(t-\sigma_{12}(t)\right)+\alpha_{13}(t) u_{3}\left(t-\sigma_{13}(t)\right)\right], \\
\dot{u}_{2}(t)=u_{2}(t)\left[\gamma_{2}(t)-\alpha_{21}(t) u_{1}\left(t-\sigma_{21}(t)\right)-\alpha_{22}(t) u_{2}\left(t-\sigma_{22}(t)\right)+\alpha_{23}(t) u_{3}\left(t-\sigma_{23}(t)\right)\right], \\
\dot{u}_{3}(t)=u_{3}(t)\left[\gamma_{3}(t)+\alpha_{31}(t) u_{1}\left(t-\sigma_{31}(t)\right)+\alpha_{32}(t) u_{2}\left(t-\sigma_{32}(t)\right)-\alpha_{33}(t) u_{3}\left(t-\sigma_{33}(t)\right)\right],
\end{array}\right.
$$

where $u_{1}(t)$ and $u_{2}(t)$ stand for the densities of competing species at time $t, u_{3}(t)$ stands for the density of cooperating species at time $t$, And $\gamma_{l}, \alpha_{l k} \in C(R,[0, \infty))$ and $\sigma_{l k} \in C(R, R)$ denote $\psi$-periodic function $(\psi>0)$. The parameter $\sigma_{l k}(t) \geq 0(l, k=1,2,3)$ is the delay. Applying the fixed point theory and constructing Lyapunov functions, $\mathrm{Lv}$ et al. [27] set up the condition to ensure the global stability of periodic solutions for model (1). For details, one can see [27].

To reveal the Hopf bifurcation nature of model (1), Xu [26] assumes that all biological and environmental coefficients remain constants and only the feedback time delays of all species $u_{i}(i=1,2,3)$ to the growth of the species themselves exist and are same [26]. Then, model (1) is rewritten as the following form:

$$
\left\{\begin{array}{l}
\dot{u}_{1}(t)=u_{1}(t)\left[\gamma_{1}-\alpha_{11} u_{1}(t-\sigma)-\alpha_{12} u_{2}(t)+\alpha_{13} u_{3}(t)\right] \\
\dot{u}_{2}(t)=u_{2}(t)\left[\gamma_{2}-\alpha_{21} u_{1}(t)-\alpha_{22} u_{2}(t-\sigma)+\alpha_{23} u_{3}(t)\right] \\
\dot{u}_{3}(t)=u_{3}(t)\left[\gamma_{3}+\alpha_{31} u_{1}(t)+\alpha_{32} u_{2}(t)-\alpha_{33} u_{3}(t-\sigma)\right]
\end{array}\right.
$$

With the Hopf bifurcation theory, normal form theory, and center manifold principle, $\mathrm{Xu}$ [26] obtained a sufficient condition to guarantee the stability behavior and the appearance for bifurcation phenomenon of model (2). Also, they have derived the concrete expression to find the nature of bifurcation periodic solution.

However, the mentioned works above are only restricted to the integer-order differential systems. Recently, the dynamics of fractional-order dynamical models has attracted great attention of many authors due to their extensive application in numerous areas, such as electromagnetic waves, medicine, mechanics, network science, biology, and finance [28-30]. Many scholars argue that fractional calculus is a powerful tool to depict real phenomena of the object world due to its owned hereditary and memory properties of various practical dynamical models [31]. In recent several decades, fractional calculus has attracted more and more attention from a large number of researchers in various fields. In particular, a great deal of interesting fruits on various dynamical natures of prey-predator systems has sprung up. For instance, Mondal et al. [32] derived the condition to ensure the stability behavior for a class of fractional-order prey-predator system; El-Saka et al. [33] analyzed the local stability and bifurcation of fractionalorder predator-prey models; Li et al. [34] dealt with the dynamical property of the solutions and global asymptotic stability for a class of fractional-order prey-predator system. For more relational publications, one can see [35-37]. Here, we particularly emphasize that the study on Hopf bifurcation for fractional-order dynamical models starts relatively late. Up to now, only a few literatures have been published. For example, Alidousti [38] investigated the bifurcation phenomenon of a fractional-order predator-prey model; Huang et al. [30] proposed a novel control technique of bifurcation for a fractional-order prey-predator model with delays; $\mathrm{Xu}$ et al. [39] revealed the effect of two delays on bifurcation for fractional-order neural networks. Xiao et al. [40] put up PD control way for Hopf bifurcations of fractional-order networks. As for more related literatures, one can see [41-44].

In terms of the above analysis, we think that it is meaningful for us to study the dynamics (especially Hopf bifurcation) of fractional-order prey-predator models. Based on the previous predator-prey model (2) and assuming that the feedback time delays of all species $u_{i}(i=1,2,3)$ to the growth of the species themselves and other species exists are same, then we propose the following fractional-order competitor-competitor-mutualist Lotka-Volterra system:

$$
\left\{\begin{array}{l}
\frac{\mathrm{d}^{\mathrm{e}} u_{1}(t)}{\mathrm{d} t^{\mathrm{Q}}}=u_{1}(t)\left[\gamma_{1}-\alpha_{11} u_{1}(t-\sigma)-\alpha_{12} u_{2}(t-\sigma)+\alpha_{13} u_{3}(t-\sigma)\right], \\
\frac{\mathrm{d}^{\mathrm{e}} u_{2}(t)}{\mathrm{d} t^{\mathrm{e}}}=u_{2}(t)\left[\gamma_{2}-\alpha_{21} u_{1}(t-\sigma)-\alpha_{22} u_{2}(t-\sigma)+\alpha_{23} u_{3}(t-\sigma)\right], \\
\frac{\mathrm{d}^{\mathrm{Q}} u_{3}(t)}{\mathrm{d} t^{\mathrm{Q}}}=u_{3}(t)\left[\gamma_{3}+\alpha_{31} u_{1}(t-\sigma)+\alpha_{32} u_{2}(t-\sigma)-\alpha_{33} u_{3}(t-\sigma)\right],
\end{array}\right.
$$

where $u_{1}(t)$ and $u_{2}(t)$ stand for the densities of competing population and $u_{3}(t)$ stands for the density of cooperating population, $\gamma_{l}, \alpha_{l k} \in C R,[0, \infty)(l, k=1,2,3)$, the parameter $\sigma \geq 0$ is the feedback time delay of different species, and $\varrho \in(0,1]$ is a constant. For more concrete meaning of coefficients for system (3), see [26, 27].

The initial condition of system (3) takes the form

$$
\begin{cases}u_{1}(\vartheta)=u_{1 \vartheta}, & -\sigma \leq \vartheta \leq t_{0} \\ u_{2}(\vartheta)=u_{2 \vartheta}, & -\sigma \leq \vartheta \leq t_{0} \\ u_{3}(\vartheta)=u_{3 \vartheta}, & -\sigma \leq \vartheta \leq t_{0}\end{cases}
$$

where $t_{0}>0$ is a constant. The key object of this work focuses on existence, uniqueness, nonnegativity, stability, and bifurcation phenomenon of model (3). Different from the methodology in $[26,27]$, in this paper, we will mainly discuss the various dynamics by applying fractional-order differential equation theory. Due to the introduction of fractional order, various dynamical behaviors of the predator-prey model (3) are different from the integer-order case. We think that it is necessary to reveal the effect of time delay, parameters of system, and fractional order on dynamics such as the stability and Hopt bifurcation. Thus, this study has significance in theory and practice.

We plan the structure of this manuscript as follows. Section 2 gives some related knowledge about fractionalorder dynamical systems. Section 3 discusses the existence, 
uniqueness, nonnegativity, local stability, and Hopf bifurcation of model (3). Section 4 gives an example to support the effectiveness of the obtained key conclusions. Section 6 ends our work.

\section{Basic Knowledge}

In this part, we present several related definitions and lemmas on fractional-order dynamical systems that will be applied in the later proof. Let $R_{+}$represent the set of all nonnegative real numbers.

Definition 1. (see [45]). Define Caputo fractional-order derivative as follows:

$$
\mathscr{D}^{\varrho} h(w)=\frac{1}{\Gamma(n-\varrho)} \int_{w_{0}}^{w} \frac{h^{(l)}(s)}{(w-s)^{w-l+1}} \mathrm{~d} s,
$$

where $h(w) \in\left(\left[w_{0}, \infty\right), R\right), \Gamma(s)=\int_{0}^{\infty} w^{s-1} e^{-w} \mathrm{~d} w, w \geq w_{0}$, and $l \in Z^{+}, l-1 \leq \varrho<l$.

The Laplace transform of Caputo fractional-order derivative is defined as follows:

$$
\begin{aligned}
\mathscr{L}\left\{\mathscr{D}^{\varrho} h(t) ; s\right\}= & s^{\varrho} \mathscr{H}(s) \\
& -\sum_{j=0}^{m-1} s^{\varrho-j-1} h^{(j)}(0), \quad m-1 \leq \varrho<m \in Z^{+},
\end{aligned}
$$

where $\mathscr{H}(s)=\mathscr{L}\{h(t)\}$. Especially, if $h^{(j)}(0)=0, j$ $=1,2, \ldots, m$, then $\mathscr{L}\left\{\mathscr{H}^{\varrho} h(t) ; s\right\}=s^{\varrho} \mathscr{H}(s)$.

Definition 2 (see [46]). $\left(v_{1 *}, v_{2 *}, v_{3 *}\right)$ is called an equilibrium point of model (3) provided that the following system

$$
\left\{\begin{array}{l}
v_{1 *}\left(\gamma_{1}-\alpha_{11} v_{1 *}-\alpha_{12} v_{2 *}+\alpha_{13} v_{3 *}\right)=0 \\
v_{2 *}\left(\gamma_{2}-\alpha_{21} v_{1 *}-\alpha_{22} v_{2 *}+\alpha_{23} v_{3 *}\right)=0 \\
v_{3 *}\left(\gamma_{3}+\alpha_{31} v_{1 *}+\alpha_{32} v_{2 *}-\alpha_{33} v_{3 *}\right)=0
\end{array}\right.
$$

holds.

Lemma 1 (see [47]). Given the following system

$$
\begin{aligned}
\frac{\mathrm{d}^{\varrho} v(t)}{\mathrm{d} t^{\varrho}} & =h(t, v(t)), \\
v\left(t_{0}\right) & =v_{t_{0}}, \quad t_{0}>0,
\end{aligned}
$$

where $\varrho \in\left(0,1 t, n h q: h\left[t_{0}, \infty\right) \times \Lambda \longrightarrow R_{+}^{n}, \Lambda \subset R_{+}^{n}\right.$. If $h(t, v)$ satisfies the local Lipschitz condition with respect to $v$, then model (3) possesses a unique solution defined on $\left[t_{0}, \infty\right)$.

Lemma 2 (see [48]). Suppose that $\varrho \in(0,1), h(t) \in C[\gamma, \delta]$ and $\mathscr{D}^{\varrho} h(t) \in C[\gamma, \delta]$, where $\gamma, \delta \in R$. If $\mathscr{D}^{\varrho} h(t)$ $\geq 0, t \in[\gamma, \delta]$, then $h(t)$ is a nondecreasing function $\forall t \in[\gamma, \delta]$. If $\mathscr{D}^{\complement} h(t) \leq 0, t \in[\gamma, \delta]$, then $h(t)$ is a nonincreasing function $\forall t \in[\gamma, \delta]$.

Lemma 3 (see $[49,50])$. For a given fractional-order model,

$$
\frac{\mathrm{d}^{\varrho} v(t)}{\mathrm{d} t^{\varrho}}=g(t, v(t)), \quad v(0)=v_{0},
$$

where $\varrho \in(0,1]$ and $g(t, v(t)): R_{+} \times R^{m} \longrightarrow R^{m}$, the equilibrium point of model (9) is locally asymptotically stable if all eigenvalues $\varsigma$ of $(\partial g(t, v) / \partial v)$ evaluated near the equilibrium point satisfy $|\arg (\varsigma)|>((\varrho \pi) / 2)$.

Lemma 4 (see [51]). For given $\$ m \$$-dimensional fractionalorder system,

$$
\left\{\begin{array}{l}
\frac{\mathrm{d}^{\mathrm{Q}_{1}} \mathscr{V}_{1}(t)}{\mathrm{d} t^{\varrho_{1}}}=l_{11} \mathscr{V}_{1}\left(t-\sigma_{11}\right)+l_{12} \mathscr{V}_{2}\left(t-\sigma_{12}\right)+\cdots+l_{1 m} \mathscr{V}_{m}\left(t-\sigma_{1 m}\right), \\
\frac{\mathrm{d}^{\mathrm{Q}_{2}} \mathscr{V}_{2}(t)}{\mathrm{d} t^{\varrho_{2}}}=l_{21} \mathscr{V}_{1}\left(t-\sigma_{21}\right)+l_{22} \mathscr{V}_{2}\left(t-\sigma_{22}\right)+\cdots+l_{2 m} \mathscr{V}_{m}\left(t-\sigma_{2 m}\right), \\
\vdots \\
\frac{\mathrm{d}^{\mathrm{Q}_{m}} \mathscr{V}_{m}(t)}{\mathrm{d} t^{\mathrm{Q}_{m}}}=l_{m 1} \mathscr{V}_{1}\left(t-\sigma_{m 1}\right)+l_{m 2} \mathscr{V}_{2}\left(t-\sigma_{m 2}\right)+\cdots+l_{m m} \mathscr{V}_{m}\left(t-\sigma_{m m}\right),
\end{array}\right.
$$

where $0<\varrho_{i}<1(i=1,2, \ldots, m)$, the initial values $\mathscr{V}_{i}(t)=\varphi_{i}(t) \in C\left[-\max _{i, l} \sigma_{i l}, 0\right]$, and $t \in\left[-\max _{i, l} \sigma_{i l}, 0\right]$, $i, l=1,2, \ldots, m$. Denote

$$
\Delta(\chi)=\left[\begin{array}{cccc}
\chi^{\varrho_{1}}-l_{11} e^{-\chi \sigma_{11}} & -l_{12} e^{-\chi \sigma_{12}} & \cdots & -l_{1 m} e^{-\chi \sigma_{1 m}} \\
-l_{21} e^{-\chi \sigma_{12}} & \chi^{\varrho_{2}}-l_{22} e^{-\chi \sigma_{22}} & \cdots & -l_{2 m} e^{-\chi \sigma_{2 m}} \\
\vdots & \vdots & \ddots & \vdots \\
-l_{m 1} e^{-\chi \sigma_{m 1}} & -l_{m 2} e^{-\chi \sigma_{m 2}} & \cdots & \chi^{\varrho_{m}}-l_{m m} e^{-\chi \sigma_{m m}}
\end{array}\right],
$$


and then, the zero solution of equation (10) is Lyapunov asymptotically stable if all roots of $\operatorname{det}(\Delta(\chi))=0$ possess negative real parts.

\section{Main Results}

\subsection{Existence and Uniqueness}

Theorem 1. Let $\Theta=\left\{\left(u_{1}, u_{2}, u_{3}\right) \in R^{3}: \max \left\{\left|u_{1}\right|,\left|u_{2}\right|\right.\right.$, $\left.\left.\left|u_{3}\right|\right\}<Q\right\}$, where $Q$ is a positive constant. Then, for every $U_{t_{0}}=$ $\left(u_{1 t_{0}}, u_{2 t_{0}}, u_{3 t_{0}}\right) \in \Theta$ and for every $t \geq t_{0}$, there exists a unique solution $U(t) \in \Theta$ of system (3) with initial value $U_{t_{0}}$.
Proof. We discuss this issue for model (3) in $\Theta \times\left[t_{0}, t^{*}\right]$, where $t^{*}<+\infty$. Let $U=\left(u_{1}, u_{2}, u_{3}\right)$ and $\widetilde{U}=\left(\widetilde{u}_{1}, \widetilde{u}_{2}, \widetilde{u}_{3}\right)$, and define the following mapping: $L(U)=\left(L_{1}(U), L_{2}(U), L_{3}(U)\right)$, where

$$
\left\{\begin{array}{l}
L_{1}(U)=u_{1}(t)\left[\gamma_{1}-\alpha_{11} u_{1}(t-\sigma)-\alpha_{12} u_{2}(t-\sigma)+\alpha_{13} u_{3}(t-\sigma)\right], \\
L_{2}[U]=u_{2}[t]\left[\gamma_{2}-\alpha_{21} u_{1}(t-\sigma)-\alpha_{22} u_{2}(t-\sigma)+\alpha_{23} u_{3}(t-\sigma)\right], \\
L_{3}(U)=u_{3}(t)\left[\gamma_{3}+\alpha_{31} u_{1}(t-\sigma)+\alpha_{32} u_{2}(t-\sigma)-\alpha_{33} u_{3}(t-\sigma)\right] .
\end{array}\right.
$$

For arbitrary $U, \widetilde{U} \in \Theta, t_{1}, t_{2} \in R, t_{1}, t_{2} \geq t_{0}$, one has

$$
\begin{aligned}
& \|L(U)-L(\widetilde{U})\| \\
& =\left|L_{1}(U)-L_{1}(\widetilde{U})\right|+\left|L_{2}(U)-L_{2}(\widetilde{U})\right|+\left|L_{3}(U)-L_{3}(\widetilde{U})\right| \\
& =\mid u_{1}\left(t_{1}\right)\left[\gamma_{1}-\alpha_{11} u_{1}\left(t_{1}-\sigma\right)-\alpha_{12} u_{2}\left(t_{1}-\sigma\right)+\alpha_{13} u_{3}\left(t_{1}-\sigma\right)\right] \\
& -u_{1}\left(t_{2}\right)\left[\gamma_{1}-\alpha_{11} u_{1}\left(t_{2}-\sigma\right)-\alpha_{12} u_{2}\left(t_{2}-\sigma\right)+\alpha_{13} u_{3}\left(t_{2}-\sigma\right)\right] \mid \\
& +\mid u_{2}\left(t_{1}\right)\left[\gamma_{2}-\alpha_{21} u_{1}\left(t_{1}-\sigma\right)-\alpha_{22} u_{2}\left(t_{1}-\sigma\right)+\alpha_{23} u_{3}\left(t_{1}-\sigma\right)\right] \\
& -u_{2}\left(t_{2}\right)\left[\gamma_{2}-\alpha_{21} u_{1}\left(t_{2}-\sigma\right)-\alpha_{22} u_{2}\left(t_{2}-\sigma\right)+\alpha_{23} u_{3}\left(t_{2}-\sigma\right)\right] \mid \\
& +\mid u_{3}\left(t_{1}\right)\left[\gamma_{3}+\alpha_{31} u_{1}\left(t_{1}-\sigma\right)+\alpha_{32} u_{2}\left(t_{1}-\sigma\right)-\alpha_{33} u_{3}\left(t_{1}-\sigma\right)\right] \\
& -u_{3}\left(t_{2}\right)\left[\gamma_{3}+\alpha_{31} u_{1}\left(t_{2}-\sigma\right)+\alpha_{32} u_{2}\left(t_{2}-\sigma\right)-\alpha_{33} u_{3}\left(t_{2}-\sigma\right)\right] \mid \\
& \leq\left|u_{1}\left(t_{1}\right)-u_{1}\left(t_{2}\right)\right|\left|\gamma_{1}-\alpha_{11} u_{1}\left(t_{1}-\sigma\right)-\alpha_{12} u_{2}\left(t_{1}-\sigma\right)+\alpha_{13} u_{3}\left(t_{1}-\sigma\right)\right| \\
& +\max \left\{\left|u_{1}\left(t_{1}\right)\right|,\left|u_{1}\left(t_{2}\right)\right|\right\} \mid\left[\gamma_{1}-\alpha_{11} u_{1}\left(t_{1}-\sigma\right)-\alpha_{12} u_{2}\left(t_{1}-\sigma\right)+\alpha_{13} u_{3}\left(t_{1}-\sigma\right)\right] \\
& -\left[\gamma_{1}-\alpha_{11} u_{1}\left(t_{2}-\sigma\right)-\alpha_{12} u_{2}\left(t_{2}-\sigma\right)+\alpha_{13} u_{3}\left(t_{2}-\sigma\right)\right] \mid \\
& +\left|u_{2}\left(t_{1}\right)-u_{2}\left(t_{2}\right)\right|\left|\gamma_{2}-\alpha_{21} u_{1}\left(t_{1}-\sigma\right)-\alpha_{22} u_{2}\left(t_{1}-\sigma\right)+\alpha_{23} u_{3}\left(t_{1}-\sigma\right)\right| \\
& +\max \left\{\left|u_{2}\left(t_{1}\right)\right|,\left|u_{2}\left(t_{2}\right)\right|\right\} \mid\left[\gamma_{2}-\alpha_{21} u_{1}\left(t_{1}-\sigma\right)-\alpha_{22} u_{2}\left(t_{1}-\sigma\right)+\alpha_{23} u_{3}\left(t_{1}-\sigma\right)\right] \\
& -\left[\gamma_{2}-\alpha_{21} u_{1}\left(t_{2}-\sigma\right)-\alpha_{22} u_{2}\left(t_{2}-\sigma\right)+\alpha_{23} u_{3}\left(t_{2}-\sigma\right)\right] \mid \\
& +\left|u_{3}\left(t_{1}\right)-u_{3}\left(t_{2}\right)\right|\left|\gamma_{3}+\alpha_{31} u_{1}\left(t_{1}-\sigma\right)+\alpha_{32} u_{2}\left(t_{1}-\sigma\right)-\alpha_{33} u_{3}\left(t_{1}-\sigma\right)\right| \\
& +\max \left\{\left|u_{3}\left(t_{1}\right)\right|,\left|u_{3}\left(t_{2}\right)\right|\right\} \mid\left[\gamma_{3}+\alpha_{31} u_{1}\left(t_{1}-\sigma\right)+\alpha_{32} u_{2}\left(t_{1}-\sigma\right)-\alpha_{33} u_{3}\left(t_{1}-\sigma\right)\right] \\
& -\left[\gamma_{3}+\alpha_{31} u_{1}\left(t_{2}-\sigma\right)+\alpha_{32} u_{2}\left(t_{2}-\sigma\right)-\alpha_{33} u_{3}\left(t_{2}-\sigma\right)\right] \mid \\
& \leq\left|u_{1}\left(t_{1}\right)-u_{1}\left(t_{2}\right)\right|\left(\gamma_{1}+\alpha_{11} Q+\alpha_{12} Q+\alpha_{13} Q\right) \\
& +\mathscr{Q} \alpha_{11}\left|u_{1}\left(t_{1}-\sigma\right)-u_{1}\left(t_{2}-\sigma\right)\right|+\mathbb{Q} \alpha_{12}\left|u_{2}\left(t_{1}-\sigma\right)-u_{2}\left(t_{2}-\sigma\right)\right| \\
& +Q \alpha_{13}\left|u_{3}\left(t_{1}-\sigma\right)-u_{3}\left(t_{2}-\sigma\right)\right| \\
& +\left|u_{2}\left(t_{1}\right)-u_{2}\left(t_{2}\right)\right|\left(\gamma_{2}+\alpha_{21} Q+\alpha_{22} Q+\alpha_{23} Q\right) \\
& +\mathbb{Q} \alpha_{21}\left|u_{1}\left(t_{1}-\sigma\right)-u_{1}\left(t_{2}-\sigma\right)\right|+Q \alpha_{22}\left|u_{2}\left(t_{1}-\sigma\right)-u_{2}\left(t_{2}-\sigma\right)\right| \\
& +Q_{23}\left|u_{3}\left(t_{1}-\sigma\right)-u_{3}\left(t_{2}-\sigma\right)\right| \\
& +\left|u_{3}\left(t_{1}\right)-u_{3}\left(t_{2}\right)\right|\left(\gamma_{3}+\alpha_{31} Q+\alpha_{32} Q+\alpha_{33} Q\right) \\
& +Q \alpha_{31}\left|u_{1}\left(t_{1}-\sigma\right)-u_{1}\left(t_{2}-\sigma\right)\right|+Q \alpha_{32}\left|u_{2}\left(t_{1}-\sigma\right)-u_{2}\left(t_{2}-\sigma\right)\right| \\
& +Q_{33}\left|u_{3}\left(t_{1}-\sigma\right)-u_{3}\left(t_{2}-\sigma\right)\right| \\
& \leq L_{1}\left|u_{1}\left(t_{1}\right)-u_{1}\left(t_{2}\right)\right|+L_{2}\left|u_{2}\left(t_{1}\right)-u_{2}\left(t_{2}\right)\right|+L_{3}\left|u_{3}\left(t_{1}\right)-u_{3}\left(t_{2}\right)\right| \text {, }
\end{aligned}
$$

where 


$$
\left\{\begin{array}{l}
L_{1}=\gamma_{1}+\mathscr{Q}\left(2 \alpha_{11}+\alpha_{21}+\alpha_{31}+\alpha_{12}+\alpha_{13}\right), \\
L_{2}=\gamma_{2}+\mathcal{Q}\left(2 \alpha_{21}+\alpha_{22}+\alpha_{32}+\alpha_{22}+\alpha_{23}\right), \\
L_{3}=\gamma_{3}+Q \mathcal{Q}\left(2 \alpha_{31}+\alpha_{23}+\alpha_{33}+\alpha_{32}+\alpha_{33}\right) .
\end{array}\right.
$$

It follows from (13) that

$$
\|L(U)-L(\widetilde{U})\| \leq L U-\widetilde{U} \mid
$$

where

$$
L=\max \left\{L_{1}, L_{2}, L_{3}\right\} .
$$

In view of Lemma 1 , one can conclude that Theorem 1 is true. We finish the proof.

3.2. Nonnegativity. Denote $\Theta_{+}=\left\{\left(u_{1}, u_{2}, u_{3}\right) \in \Theta: u_{1}, u_{2}\right.$, $\left.u_{3} \in R_{+}\right\}$.

Theorem 2. Each solution of model (3) that begins with $\Theta_{+}$ remains nonnegative.

Proof. Our object is to prove that $u_{i}(t) \geq 0(i=1,2,3) \forall t \geq t_{0}$. Let $U\left(t_{0}\right)=\left(u_{1}\left(t_{0}\right), u_{1}\left(t_{0}\right), u_{1}\left(t_{0}\right)\right) \in \Theta_{+}$be the initial value of system (3). Assume that $\exists$ is a constant and $t_{0} \leq t<\rho$ such that

$$
\left\{\begin{array}{l}
u_{i}(t)=0, \quad t_{0} \leq t<\rho, \\
u_{i}(\rho)=0, \\
u_{i}\left(\rho^{+}\right)<0,
\end{array}\right.
$$

where $i=1,2,3$. In view of system (3), one has

$$
\left\{\begin{array}{l}
\left.\frac{\mathrm{d}^{\varrho} u_{1}(t)}{\mathrm{d} t^{\varrho}}\right|_{u_{i}=0}=0 \\
\left.\frac{\mathrm{d}^{\varrho} u_{2}(t)}{\mathrm{d} t^{\varrho}}\right|_{u_{i}=0}=0 \\
\left.\frac{\mathrm{d}^{\varrho} u_{3}(t)}{\mathrm{d} t^{\varrho}}\right|_{u_{i}=0}=0
\end{array}\right.
$$

where $i=1,2,3$. By Lemma 2 , one knows that $u_{i}\left(\rho^{+}\right)=0(i=1,2,3)$, which contradicts $u_{i}\left(\rho^{+}\right)<0(i=$ $1,2,3)$ in (17). Thus, one can conclude that $u_{i}(t) \geq 0 \forall t \geq t_{0}$. The proof is complete.

3.3. Stability and Hopf Bifurcation. In the section, we shall focus on the local stability and the appearance of Hopf bifurcation of system (3). Consider the biological implication of system (3); we only seek the sufficient condition to guarantee the local stability of the positive equilibrium and the emergence of Hopf bifurcations of system (3).

Clearly, system (3) has a unique positive equilibrium $U_{0}\left(u_{1}^{*}, u_{2}^{*}, u_{3}^{*}\right)$ provided that the following condition

$$
\left(\mathscr{M}_{1}\right) \operatorname{sign}\{\mathscr{A}\}=\operatorname{sign}\left\{\mathscr{A}_{1}\right\}=\operatorname{sign}\left\{\mathscr{A}_{2}\right\}=\operatorname{sign}\left\{\mathscr{A}_{3}\right\},
$$

is satisfied, where

$$
\left\{\begin{array}{l}
\mathscr{A}=\operatorname{det}\left[\begin{array}{lll}
\alpha_{11} \alpha_{12}-\alpha_{13} & \alpha_{21} \alpha_{22}-\alpha_{23} & \alpha_{31} \alpha_{31}-\alpha_{33}
\end{array}\right] \neq 0, \\
\mathscr{A}_{1}=\operatorname{det}\left[\begin{array}{ccc}
\gamma_{1} & \alpha_{12} & -\alpha_{13} \\
\gamma_{2} & \alpha_{22} & -\alpha_{23} \\
-\gamma_{3} & \alpha_{31} & -\alpha_{33}
\end{array}\right], \\
\mathscr{A}_{2}=\operatorname{det}\left[\begin{array}{lll}
\alpha_{11} & \gamma_{1} & -\alpha_{13} \\
\alpha_{21} & \gamma_{2} & -\alpha_{23} \\
\alpha_{31} & -\gamma_{3} & -\alpha_{33}
\end{array}\right], \\
\mathscr{A}_{3}=\operatorname{det}\left[\begin{array}{lll}
\alpha_{11} & \alpha_{12} & \gamma_{1} \\
\alpha_{21} & \alpha_{22} & \gamma_{2} \\
\alpha_{31} & \alpha_{31} & -\gamma_{3}
\end{array}\right], \\
u_{1}^{*}=\frac{\mathscr{A}_{1}}{\mathscr{A}}, \\
u_{2}^{*}=\frac{\mathscr{A}_{2}}{\mathscr{A}}, \\
u_{3}^{*}=\frac{\mathscr{A}_{3}}{\mathscr{A}} .
\end{array}\right.
$$
takes

The linear system of equation (3) near $U_{0}\left(u_{1}^{*}, u_{2}^{*}, u_{3}^{*}\right)$

$$
\left\{\begin{array}{l}
\frac{\mathrm{d}^{\mathrm{Q}} u_{1}(t)}{\mathrm{d} t^{\varrho}}=a_{11} u_{1}(t-\sigma)+a_{12} u_{2}(t-\sigma)+a_{13} u_{3}(t-\sigma), \\
\frac{\mathrm{d}^{\mathrm{Q}} u_{2}(t)}{\mathrm{d} t^{\varrho}}=a_{21} u_{1}(t-\sigma)+a_{22} u_{2}(t-\sigma)+a_{23} u_{3}(t-\sigma), \\
\frac{\mathrm{d}^{\mathrm{Q}} u_{3}(t)}{\mathrm{d} t^{\varrho}}=a_{31} u_{1}(t-\sigma)+a_{32} u_{2}(t-\sigma)+a_{33} u_{3}(t-\sigma),
\end{array}\right.
$$

where

$$
\left\{\begin{array}{l}
a_{11}=-u_{1 *} \alpha_{11}, a_{12}=-u_{1 *} \alpha_{12}, a_{13}=u_{1 *} \alpha_{13} \\
a_{21}=-u_{2 *} \alpha_{21}, a_{22}=-u_{2 *} \alpha_{22}, a_{23}=u_{2 *} \alpha_{23} \\
a_{31}=u_{3 *} \alpha_{31}, a_{32}=u_{3 *} \alpha_{32}, a_{33}=-u_{3 *} \alpha_{33} .
\end{array}\right.
$$

The characteristic equation of equation (21) is computed as follows: 


$$
\operatorname{det}\left[\begin{array}{ccc}
s^{\varrho}-a_{11} e^{-s \sigma} & -a_{12} e^{-s \sigma} & -a_{13} e^{-s \sigma} \\
-a_{21} e^{-s \sigma} & s^{\varrho}-a_{22} e^{-s \sigma} & -a_{23} e^{-s \sigma} \\
-a_{31} e^{-s \sigma} & -a_{32} e^{-s \sigma} & s^{\varrho}-a_{33} e^{-s \sigma}
\end{array}\right]=0
$$

which leads to

$$
s^{3 \varrho}+b_{1} s^{2 \varrho} e^{-s \sigma}+b_{2} s^{\varrho} e^{-2 s \sigma}+b_{3} e^{-3 s \sigma}=0
$$

where

$$
\left\{\begin{array}{l}
b_{1}=-\left(a_{11}+a_{22}+a_{33}\right), \\
b_{2}=a_{11} a_{33}+a_{22} a_{33}+a_{11} a_{22}-a_{13} a_{31} \\
-a_{12} a_{21}-a_{23} a_{32}, \\
b_{3}=a_{13} a_{22} a_{31}+a_{12} a_{21} a_{33}+a_{11} a_{23} a_{32} \\
-a_{11} a_{22} a_{33}-a_{13} a_{21} a_{32} .
\end{array}\right.
$$

In terms of (24), we have

$$
s^{3 \varrho} e^{2 s \sigma}+b_{1} s^{2 \varrho} e^{s \sigma}+b_{2} s^{\varrho}+b_{3} e^{-s \sigma}=0 .
$$

Let $s=i \varphi=\varphi(\cos (\pi / 2)+i \sin (\pi / 2))$ be the root of $(26)$; then, one has

$$
\left\{\begin{array}{l}
\mathscr{G}_{1}(\varrho) \cos 2 \varphi \sigma+\mathscr{G}_{2}(\varrho) \sin 2 \varphi \sigma+\mathscr{G}_{3}(\varrho) \cos \varphi \sigma+\mathscr{G}_{4}(\varrho) \sin \varphi \sigma=0, \\
\mathcal{S}_{1}(\varrho) \cos 2 \varphi \sigma+\mathcal{S}_{2}(\varrho) \sin 2 \varphi \sigma+\mathcal{S}_{3}(\varrho) \cos \varphi \sigma+\mathcal{S}_{4}(\varrho) \sin \varphi \sigma=0,
\end{array}\right.
$$

where

$$
\left\{\begin{array}{l}
\mathscr{G}_{1}(\varrho)=\varphi^{3 \varrho} \cos \frac{3 \varrho \pi}{2}+b_{2} \varphi^{\varrho} \cos \frac{\varrho \pi}{2}, \\
\mathscr{G}_{2}(\varrho)=b_{2} \varphi^{\varrho} \sin \frac{\varrho \pi}{2}-\varphi^{3 \varrho} \sin \frac{3 \varrho \pi}{2} \\
\mathscr{G}_{3}(\varrho)=b_{1} \varphi^{2 \varrho} \cos \varrho \pi+b_{3}, \\
\mathscr{G}_{4}(\varrho)=-b_{1} \varphi^{2 \varrho} \sin \varrho \pi, \\
\mathcal{S}_{1}(\varrho)=\varphi^{3 \varrho} \sin \frac{3 \varrho \pi}{2}+b_{2} \varphi^{\varrho} \sin \frac{\varrho \pi}{2} \\
\mathcal{S}_{2}(\varrho)=\varphi^{3 \varrho} \cos \frac{3 \varrho \pi}{2}-b_{2} \varphi^{\varrho} \cos \frac{\varrho \pi}{2} \\
\mathcal{S}_{3}(\varrho)=b_{1} \varphi^{2 \varrho} \sin \varrho \pi, \\
\mathcal{S}_{4}(\varrho)=b_{1} \varphi^{2 \varrho} \cos \varrho \pi-b_{3} .
\end{array}\right.
$$

In (28), let

$$
\left\{\begin{array}{l}
c_{1}=\cos \frac{3 \varrho \pi}{2}, \\
c_{2}=b_{2} \cos \frac{\varrho \pi}{2}, \\
c_{3}=b_{2} \sin \frac{\varrho \pi}{2}, \\
c_{4}=-\sin \frac{3 \varrho \pi}{2}, \\
c_{5}=b_{1} \cos \varrho \pi, \\
c_{6}=b_{3}, \\
c_{13}=b_{1} \cos \varrho \pi \\
c_{7}=-b_{1} \sin \varrho \pi \\
c_{10}=\cos \frac{3 \varrho \pi}{2}, \\
c_{11}=-b_{2} \cos \frac{\varrho \pi}{2} \\
c_{8}=b_{2} \sin \frac{3 \varrho \pi}{2},
\end{array}\right.
$$

and then, (28) takes the form:

$$
\left\{\begin{array}{l}
\mathscr{G}_{1}(\varrho)=c_{1} \varphi^{3 \varrho}+c_{2} \varphi^{\varrho}, \\
\mathscr{G}_{2}(\varrho)=c_{3} \varphi^{\varrho}+c_{4} \varphi^{3 \varrho}, \\
\mathscr{G}_{3}(\varrho)=c_{5} \varphi^{2 \varrho}+c_{6}, \\
\mathscr{G}_{4}(\varrho)=c_{7} \varphi^{2 \varrho}, \\
\mathcal{S}_{1}(\varrho)=c_{8} \varphi^{3 \varrho}+c_{9} \varphi^{\varrho}, \\
\mathcal{S}_{2}(\varrho)=c_{10} \varphi^{3 \varrho}+c_{11} \varphi^{\varrho}, \\
\mathcal{S}_{3}(\varrho)=c_{12} \varphi^{2 \varrho}, \\
\mathcal{S}_{4}(\varrho)=c_{13} \varphi^{2 \varrho}+c_{14} .
\end{array}\right.
$$

For (27), we consider two cases.

Case 1. If $\sin \varphi \sigma=\sqrt{1-\cos ^{2} \varphi \sigma}$, then the first equation of (27) becomes 


$$
\begin{aligned}
& \mathscr{G}_{1}(\varrho)\left(2 \cos ^{2} \varphi \sigma-1\right)+2 \mathscr{G}_{2}(\varrho) \cos \varphi \sigma \sqrt{1-\cos ^{2} \varphi \sigma} \\
& +\mathscr{G}_{3}(\varrho) \cos \varphi \sigma+\mathscr{G}_{4}(\varrho) \sqrt{1-\cos ^{2} \varphi \sigma}=0 .
\end{aligned}
$$

Hence,

$$
\begin{aligned}
& {\left[2 \mathscr{G}_{2}(\varrho) \cos \varphi \sigma \sqrt{1-\cos ^{2} \varphi \sigma}+\mathscr{G}_{4}(\varrho) \sqrt{1-\cos ^{2} \varphi \sigma}\right]^{2}} \\
& =\left[\mathscr{G}_{1}(\varrho)\left(2 \cos ^{2} \varphi \sigma-1\right)+\mathscr{G}_{3}(\varrho) \cos \varphi \sigma\right]^{2}
\end{aligned}
$$

which leads to

$$
\vartheta_{1} \cos ^{4} \varphi \sigma+\vartheta_{2} \cos ^{3} \varphi \sigma+\vartheta_{3} \cos ^{2} \varphi \sigma+\vartheta_{4} \cos \varphi \sigma+\vartheta_{5}=0,
$$

where

$$
\left\{\begin{array}{l}
\vartheta_{1}=4 \mathscr{G}_{1}^{2}(\varrho)+4 \mathscr{G}_{2}^{2}(\varrho) \\
\vartheta_{2}=4 \mathscr{G}_{1}(\varrho) \mathscr{G}_{3}(\varrho)+4 \mathscr{G}_{2}(\varrho) \mathscr{G}_{4}(\varrho) \\
\vartheta_{3}=\mathscr{G}_{3}^{2}(\varrho)+\mathscr{G}_{4}^{2}(\varrho)-4 \mathscr{G}_{1}^{2}(\varrho)-4 \mathscr{G}_{2}^{2}(\varrho) \\
\vartheta_{4}=-\left[2 \mathscr{G}_{1}(\varrho) \mathscr{G}_{3}(\varrho)+4 \mathscr{G}_{2}(\varrho) \mathscr{G}_{4}(\varrho)\right] \\
\vartheta_{5}=\mathscr{G}_{1}^{2}(\varrho)-\mathscr{G}_{4}^{2}(\varrho)
\end{array}\right.
$$

Set $\cos \varphi \sigma=r$, and let

$$
z(r)=r^{4}+\frac{\vartheta_{2}}{\vartheta_{1}} r^{3}+\frac{\vartheta_{3}}{\vartheta_{1}} r^{2}+\frac{\vartheta_{4}}{\vartheta_{1}} r+\frac{\vartheta_{5}}{\vartheta_{1}} .
$$

Hence,

$$
\frac{\mathrm{d} z(r)}{\mathrm{d} r}=4 r^{3}+\frac{3 \vartheta_{2}}{\vartheta_{1}} r^{2}+\frac{2 \vartheta_{3}}{\vartheta_{1}} r+\frac{\vartheta_{4}}{\vartheta_{1}}
$$

Denote

$$
r^{3}+\frac{3 \vartheta_{2}}{\vartheta_{1}} r^{2}+\frac{2 \vartheta_{3}}{\vartheta_{1}} r+\frac{\vartheta_{4}}{\vartheta_{1}}=0 .
$$

Set $p=r+\left(\vartheta_{2} / 4 \vartheta_{1}\right)$, and then, (37) takes the form

$$
p^{3}+f_{1} p+f_{2}=0
$$

where

$$
\begin{aligned}
& f_{1}=\frac{\vartheta_{3}}{2 \vartheta_{1}}-\frac{3 \vartheta_{2}^{2}}{16 \vartheta_{1}^{2}}, \\
& f_{2}=\frac{\vartheta_{2}^{3}}{32 \vartheta_{1}^{3}}-\frac{\vartheta_{2} \vartheta_{3}}{8 \vartheta_{1}^{2}}+\frac{\vartheta_{4}}{4 \vartheta_{1}} .
\end{aligned}
$$

Let

$$
\begin{aligned}
& q_{1}=\left(\frac{f_{2}}{2}\right)^{2}+\left(\frac{f_{1}}{3}\right)^{3} \\
& q_{2}=\frac{-1+i \sqrt{3}}{2}
\end{aligned}
$$

It follows from (38) that

$$
\left\{\begin{array}{l}
p_{1}=\sqrt[3]{-\frac{f_{2}}{2}+\sqrt{q_{1}}}+\sqrt[3]{-\frac{f_{2}}{2}-\sqrt{q_{1}}} \\
p_{2}=\sqrt[3]{-\frac{f_{2}}{2}+\sqrt{q_{1}} q_{2}}+\sqrt[3]{-\frac{f_{2}}{2}-\sqrt{q_{1}} q_{2}^{2}} \\
p_{3}=\sqrt[3]{-\frac{f_{2}}{2}+\sqrt{q_{1}} q_{2}^{2}}+\sqrt[3]{-\frac{f_{2}}{2}-\sqrt{q_{1}} q_{2}}
\end{array}\right.
$$

According to the discussion above, one easily obtains the expression of $\cos \varphi \sigma$. Then, one easily gets the expression of $\sin \varphi \sigma$. Here, we suppose that

$$
\begin{aligned}
\cos \varphi \sigma & =g_{1}(\varphi), \\
\sin \varphi \sigma & =g_{2}(\varphi) .
\end{aligned}
$$

Then,

$$
g_{1}^{2}(\varphi)+g_{2}^{2}(\varphi)=1
$$

With the aid of Matlab 7.0, one can get the root (say $\varphi$ ) of equation (43). Then, one obtains

$$
\sigma^{1 k}=\frac{1}{\varphi}\left[\arccos g_{1}(\varphi)+2 k \pi\right], \quad k=0,1,2, \ldots
$$

Case 2. If $\sin \varphi \sigma=-\sqrt{1-\cos ^{2} \varphi \sigma}$, applying the same way to this case, we can obtain

$$
\begin{aligned}
\cos \varphi \sigma & =h_{1}(\varphi), \\
\sin \varphi \sigma & =h_{2}(\varphi) .
\end{aligned}
$$

Then,

$$
h_{1}^{2}(\varphi)+h_{2}^{2}(\varphi)=1
$$

By Matlab 7.0, one can obtain the root (say $\varphi$ ) of equation (46). So,

$$
\sigma^{2 k}=\frac{1}{\varphi}\left[\arccos h_{1}(\varphi)+2 k \pi\right], \quad k=0,1,2, \ldots
$$

Set

$$
\sigma_{0}=\min \left\{\sigma^{1 k}, \sigma^{2 k}\right\}, \quad k=0,1,2, \ldots
$$

In the sequel, we check the transversality condition of the appearance of Hopf bifurcation. We give the hypothesis as follows: $\left(\mathscr{M}_{2}\right) \mathscr{E}_{11} \mathscr{E}_{21}+\mathscr{E}_{12} \mathscr{E}_{22}>0$, where 


$$
\begin{aligned}
& \mathscr{Z}_{11}=3 \varrho \varphi_{0}^{3 \varrho-1}\left[\cos \frac{(3 \varrho-1) \pi}{2} \cos 2 \varphi_{0} \sigma_{0}-\sin \frac{(3 \varrho-1) \pi}{2} \sin 2 \varphi_{0} \sigma_{0}\right] \\
& +2 \varphi_{0}^{3 \varrho} \sigma_{0}\left[\cos \frac{3 \varrho \pi}{2} \cos 2 \varphi_{0} \sigma_{0}-\sin \frac{3 \varrho \pi}{2} \sin 2 \varphi_{0} \sigma_{0}\right] \\
& +2 b_{1} \varrho \varphi_{0}^{2 \varrho-1}\left[\cos \frac{(2 \varrho-1) \pi}{2} \cos \varphi_{0} \sigma_{0}-\sin \frac{(2 \varrho-1) \pi}{2} \sin \varphi_{0} \sigma_{0}\right] \\
& +b_{1} \sigma_{0} \varphi_{0}^{2 \varrho}\left[\cos \varrho \pi \cos \varphi_{0} \sigma_{0}-\sin \varrho \pi \sin \varphi_{0} \sigma_{0}\right] \\
& +b_{2} \varrho \varphi_{0}^{\varrho-1} \cos \frac{(\varrho-1) \pi}{2}-b_{3} \sigma_{0} \cos \varphi_{0} \sigma_{0} \\
& \mathscr{Z}_{12}=3 \varrho \varphi_{0}^{3 \varrho-1}\left[\cos \frac{(3 \varrho-1) \pi}{2} \sin 2 \varphi_{0} \sigma_{0}+\sin \frac{(3 \varrho-1) \pi}{2} \cos 2 \varphi_{0} \sigma_{0}\right] \\
& +2 \varphi_{0}^{3 \varrho} \sigma_{0}\left[\cos \frac{3 \varrho \pi}{2} \sin 2 \varphi_{0} \sigma_{0}+\sin \frac{3 \varrho \pi}{2} \cos 2 \varphi_{0} \sigma_{0}\right] \\
& +2 b_{1} \varrho \varphi_{0}^{2 \varrho-1}\left[\cos \frac{(2 \varrho-1) \pi}{2} \sin \varphi_{0} \sigma_{0}+\sin \frac{(2 \varrho-1) \pi}{2} \cos \varphi_{0} \sigma_{0}\right] \\
& +b_{1} \sigma_{0} \varphi_{0}^{2 \varrho}\left[\cos \varrho \pi \sin \varphi_{0} \sigma_{0}+\sin \varrho \pi \cos \varphi_{0} \sigma_{0}\right] \\
& +b_{2} \varrho \varphi_{0}^{\varrho-1} \sin \frac{(\varrho-1) \pi}{2}+b_{3} \sigma_{0} \sin \varphi_{0} \sigma_{0} \\
& \mathscr{Z}_{21}=b_{3} \varphi_{0} \sin \varphi_{0} \sigma_{0}-2 \varphi_{0}^{3 \varrho+1}\left[\cos \frac{(3 \varrho+1) \pi}{2} \cos 2 \varphi_{0} \sigma_{0}-\sin \frac{(3 \varrho+1) \pi}{2} \sin 2 \varphi_{0} \sigma_{0}\right] \\
& -2\left(b_{2} \varrho_{0}^{2 \sigma} \sin \sigma \pi+b_{1} \varrho_{0}^{\sigma} \sin \frac{\sigma \pi}{2}\right) \varrho_{0} \cos 2 \varrho_{0} \eta_{0} \\
& -b_{1} \varphi_{0}^{2 \varrho+1}\left[\cos \frac{(2 \varrho+1) \pi}{2} \cos 2 \varphi_{0} \sigma_{0}-\sin \frac{(2 \varrho+1) \pi}{2} \sin 2 \varphi_{0} \sigma_{0}\right], \\
& \mathscr{Z}_{22}=b_{3} \varphi_{0} \cos \varphi_{0} \sigma_{0}-2 \varphi_{0}^{3 \varrho+1}\left[\cos \frac{(3 \varrho+1) \pi}{2} \sin 2 \varphi_{0} \sigma_{0}+\sin \frac{(3 \varrho+1) \pi}{2} \cos 2 \varphi_{0} \sigma_{0}\right] \\
& -b_{1} \varphi_{0}^{2 \varrho+1}\left[\cos \frac{(2 \varrho+1) \pi}{2} \sin 2 \varphi_{0} \sigma_{0}+\sin \frac{(2 \varrho+1) \pi}{2} \cos 2 \varphi_{0} \sigma_{0}\right]
\end{aligned}
$$


Lemma 5. Suppose that $s(\sigma)=\xi(\sigma)+i \eta(\sigma)$ is a root of equation (26) near $\sigma=\sigma_{0}$ such that $\xi\left(\sigma_{0}\right)=0, \eta\left(\sigma_{0}\right)=\varphi_{0}$; then, one has $\left.\operatorname{Re}[d s / d \sigma]\right|_{\sigma=\sigma_{0}, \varphi=\varphi_{0}}>0$.
Proof. In terms of equation (26), one knows that

$$
\begin{aligned}
& 3 \varrho s^{3 \varrho-1} e^{2 s \sigma} \frac{\mathrm{d} s}{\mathrm{~d} \sigma}+2 s^{3 \varrho} e^{2 s \sigma}\left(\frac{\mathrm{d} s}{\mathrm{~d} \sigma} \sigma+s\right) \\
& +2 b_{1} \varrho s^{2 \varrho-1} e^{s \sigma} \frac{\mathrm{d} s}{\mathrm{~d} \sigma}+b_{1} e^{s \sigma} s^{2 \varrho}\left(\frac{\mathrm{d} s}{\mathrm{~d} \sigma} \sigma+s\right)+b_{2} \varrho s^{\varrho-1} \frac{\mathrm{d} s}{\mathrm{~d} \sigma}-b_{3} e^{-s \sigma}\left(\frac{\mathrm{d} s}{\mathrm{~d} \sigma} \sigma+s\right)=0
\end{aligned}
$$

which leads to

$$
\begin{aligned}
& {\left[3 \varrho s^{3 \varrho-1} e^{2 s \sigma}+2 s^{3 \varrho} e^{2 s \sigma} \sigma+2 b_{1} \varrho s^{2 \varrho-1} e^{s \sigma}\right.} \\
& \left.\quad+b_{1} e^{s \sigma} s^{2 \varrho} \sigma+b_{2} \varrho s^{\varrho-1}-b_{3} e^{-s \sigma} \sigma\right] \frac{\mathrm{d} s}{\mathrm{~d} \sigma} \\
& =b_{3} s e^{-s \sigma}-2 s^{3 \varrho+1} e^{2 s \sigma}-b_{1} s^{2 \varrho+1} e^{s \sigma} .
\end{aligned}
$$

Then,

$$
\left[\frac{\mathrm{d} s}{\mathrm{~d} \sigma}\right]^{-1}=\frac{\mathscr{Z}_{1}(s)}{\mathscr{E}_{2}(s)}-\frac{\sigma}{s},
$$

where

$$
\left\{\begin{array}{l}
\mathscr{Z}_{1}(s)=3 \varrho s^{3 \varrho-1} e^{2 s \sigma}+2 s^{3 \varrho} e^{2 s \sigma} \sigma+2 b_{1} \varrho s^{2 \varrho-1} e^{s \sigma} \\
+b_{1} e^{s \sigma} s^{2 \varrho} \sigma+b_{2} \varrho s^{\varrho-1}-b_{3} e^{-s \sigma} \sigma, \\
\mathscr{Z}_{2}(s)=b_{3} s e^{-s \sigma}-2 s^{3 \varrho+1} e^{2 s \sigma}-b_{1} s^{2 \varrho+1} e^{s \sigma} .
\end{array}\right.
$$

So,

$\left.\operatorname{Re}\left\{\frac{\mathrm{d} s}{\mathrm{~d} \sigma}\right\}\right|_{\sigma=\sigma_{0}, \varphi=\varphi_{0}}=\left.\operatorname{Re}\left\{\frac{Z_{1}(s)}{Z_{2}(s)}\right\}\right|_{\sigma=\sigma_{0}, \varphi=\varphi_{0}}=\frac{\mathscr{Z}_{11} \mathscr{Z}_{21}+\mathscr{Z}_{12} \mathscr{Z}_{22}}{\mathscr{Z}_{21}^{2}+\mathscr{Z}_{22}^{2}}$.

By $\left(\mathscr{M}_{2}\right)$, one obtains

$$
\left.\operatorname{Re}\left\{\left[\frac{\mathrm{d} s}{\mathrm{~d} \sigma}\right]^{-1}\right\}\right|_{\sigma=\sigma_{0}, \varphi=\varphi_{0}}>0 .
$$

This finishes the proof of Lemma 5.

Suppose that

$$
\begin{gathered}
\left(\mathscr{M}_{3}\right) b_{1}>0, \\
b_{1} b_{2}>b_{3}, \\
b_{3}>0 .
\end{gathered}
$$

Lemma 6. Provided that $\sigma=0$ and $\left(\mathscr{M}_{3}\right)$ is fulfilled, then system (3) is locally asymptotically stable.
Proof. If $\sigma=0$, then (24) takes the form

$$
\lambda^{3}+b_{1} \lambda^{2}+b_{2} \lambda+b_{3}=0
$$

By $\left(\mathscr{M}_{3}\right)$, one knows that all roots $\lambda_{i}$ of (57) satisfy $\left|\arg \left(\lambda_{i}\right)\right|>((\varrho \pi) / 2)(i=1,2,3,4)$. Thus, one knows that Lemma 6 is right. The proof finishes.

Based on the investigation above, we have the following conclusion.

Theorem 3. Provided that $\left(\mathscr{M}_{1}\right)-\left(\mathscr{M}_{3}\right)$ hold true, then $U_{0}\left(u_{1 *}, u_{1 *}, u_{3 *}\right)$ of system (3) is locally asymptotically stable if $\sigma \in\left[0, \sigma_{0}\right)$, and a Hopf bifurcation exists near $U_{0}\left(u_{1 *}, u_{1 *}, u_{3 *}\right)$ if $\sigma=\sigma_{0}$.

\section{Computer Simulations}

In this paper, we apply implicit Euler's scheme which is introduced in [52] to carry our numerical simulations. Given the following fractional-order predator-prey model

$$
\left\{\begin{array}{l}
\frac{\mathrm{d}^{\varrho} u_{1}(t)}{\mathrm{d} t^{\varrho}}=u_{1}(t)\left[8-3 u_{1}(t-\sigma)-2 u_{2}(t-\sigma)+0.3 u_{3}(t-\sigma)\right], \\
\frac{\mathrm{d}^{\varrho} u_{2}(t)}{\mathrm{d} t^{\varrho}}=u_{2}(t)\left[7-2 u_{1}(t-\sigma)-2 u_{2}(t-\sigma)+0.3 u_{3}(t-\sigma)\right], \\
\frac{\mathrm{d}^{\varrho} u_{3}(t)}{\mathrm{d} t^{\varrho}}=u_{3}(t)\left[6+0.2 u_{1}(t-\sigma)+0.2 u_{2}(t-\sigma)-3 u_{3}(t-\sigma)\right],
\end{array}\right.
$$

we can easily know that model (58) possesses the equilibrium point $U_{0}(1.0000,2.8384,2.2559)$. Let $\varrho=0.79$. Then, $r_{1}=8, \gamma_{2}=7, \gamma_{3}=6, \alpha_{11}=3, \alpha_{12}=2, \alpha_{13}=0.3, \quad \alpha_{21}=2$, $\alpha_{22}=2, \alpha_{23}=0.3, \alpha_{31}=0.2, \alpha_{32}=0.2$, and $\alpha_{33}=3$. Hence, $a_{11}=-3, a_{12}=-2, a_{13}=0.3, a_{21}=-5.6788, a_{22}=-5.6788$, , $a_{23}=0.6768, a_{31}=0.4512, a_{32}=0.4512, a_{33}=-6.7677, \varphi_{0}=$ 0.3127 , and $\sigma_{0}=0.192$. Then, $b_{1}=15.4465, b_{2}=113.7636$, $b_{3}=151.8836, \mathscr{Z}_{11}=0.3742, \mathscr{Z}_{12}=0.6425, \mathscr{Z}_{21}=0.5004$, and $\mathscr{Z}_{22}=0.7157$. Thus, the hypothesis $\left(\mathscr{M}_{1}\right)-\left(\mathscr{M}_{3}\right)$ of 


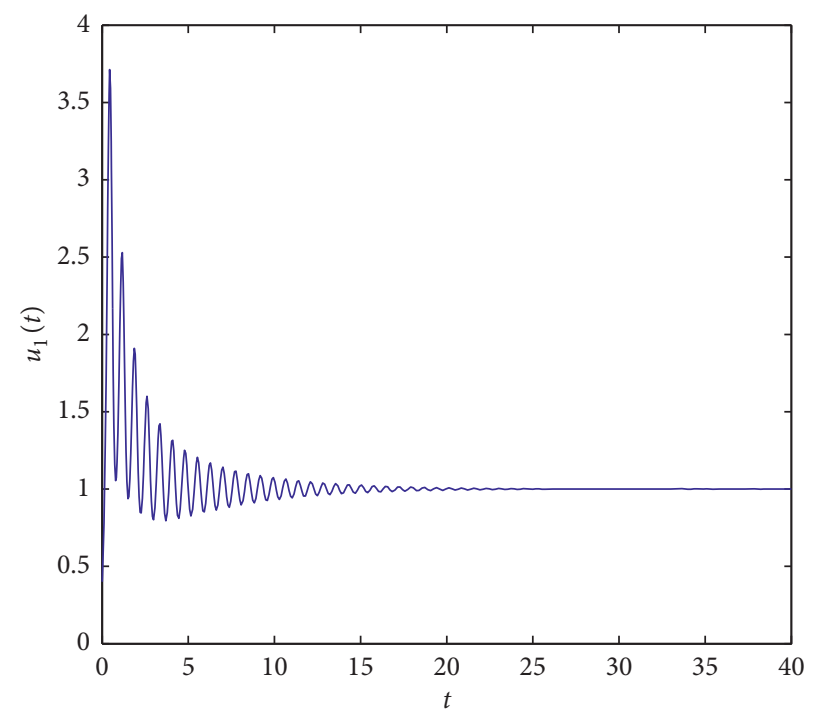

Figure 1: State variable $u_{1}(t)$ versus time $t$ of system (58) when $\sigma=0.18<\sigma_{0}=0.192$.

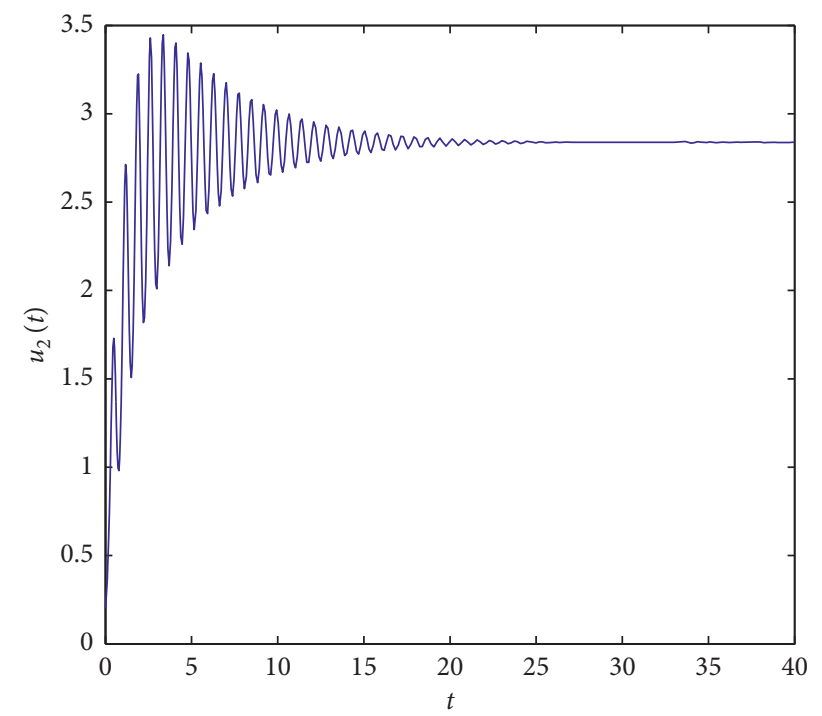

FIGURE 2: . State variable $u_{2}(t)$ versus time $t$ of system (58) when $\sigma=0.18<\sigma_{0}=0.192$.

Theorem 3 hold true. If $\sigma \in[0,0.192)$, the positive equilibrium point $U_{0}(1.0000,2.8384,2.2559)$ of system (58) is locally asymptotically stable. For this case, we choose $\sigma=0.18<$ $\sigma_{0}=0.192$. The simulation plots are displayed in Figures 1-3. Figures 1-3 reveal that if $\sigma$ is less than $\sigma_{0}=0.192$, then the densities of competing species $u_{1}$ and $u_{2}$ of system (58) will be tardily close to $1.0000,2.8384$, respectively, and the density of cooperating species $u_{3}$ of system (58) will be tardily close to 2.2559. When $\sigma \in[0.192,+\infty)$, then system (58) loses its stability and Hopf bifurcation behavior emerges. For this case, we choose $\sigma=0.33$. The numerical simulation diagrams are presented in Figures 4-6. Figures 4-6 confirm that when $\sigma$ is greater than $\sigma_{0}=0.192$, then the densities of competing species $u_{1}$ and $u_{2}$ and the density of cooperating species $u_{3}$ will remain 


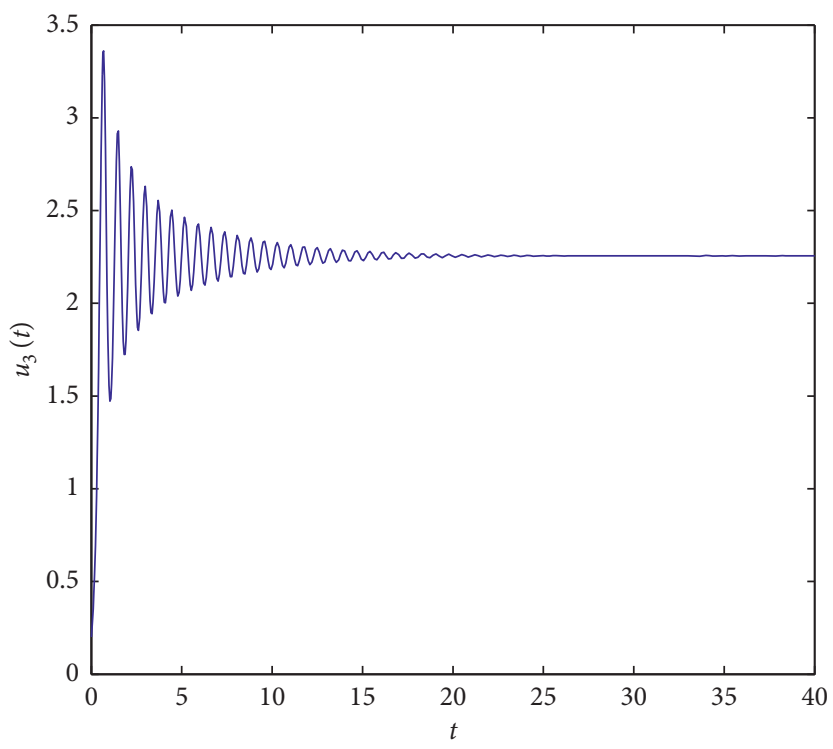

Figure 3: State variable $u_{3}(t)$ versus time $t$ of system (58) when $\sigma=0.18<\sigma_{0}=0.192$.

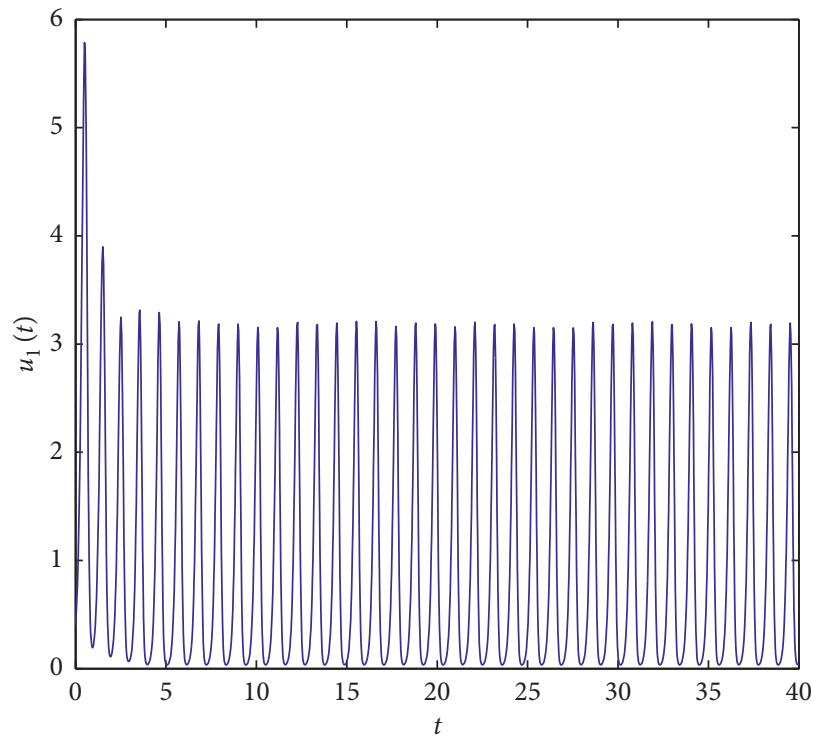

FIgURE 4: State variable $u_{1}(t)$ versus time $t$ when $\sigma=0.33>\sigma_{0}=0.192$.

periodic motion around the positive equilibrium point $U_{0}(1.0000,2.8384,2.2559)$, namely, Hopf bifurcation phenomenon takes place around $U_{0}(1.0000,2.8384,2.2559)$. In order to illustrate the bifurcation phenomenon intuitively, we plot the bifurcation diagrams (see Figures 7-9). From Figures 7-9, we can easily see that the bifurcation value is 0.192 . In addition, we give the relationship table between $\varphi_{0}$ and $\sigma_{0}$ in Table 1. 


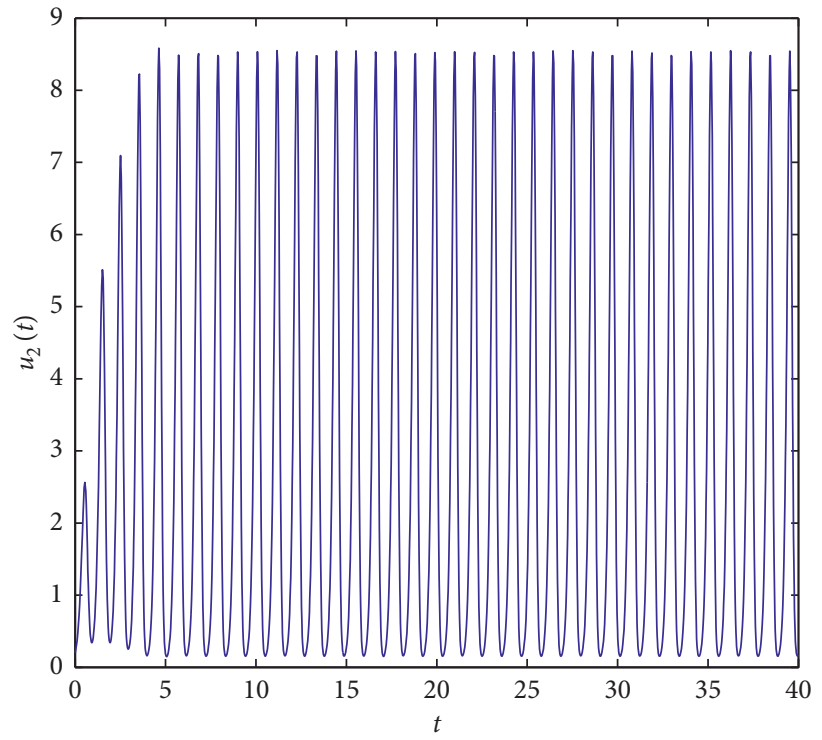

Figure 5: State variable $u_{2}(t)$ versus time $t$ when $\sigma=0.33>\sigma_{0}=0.192$.

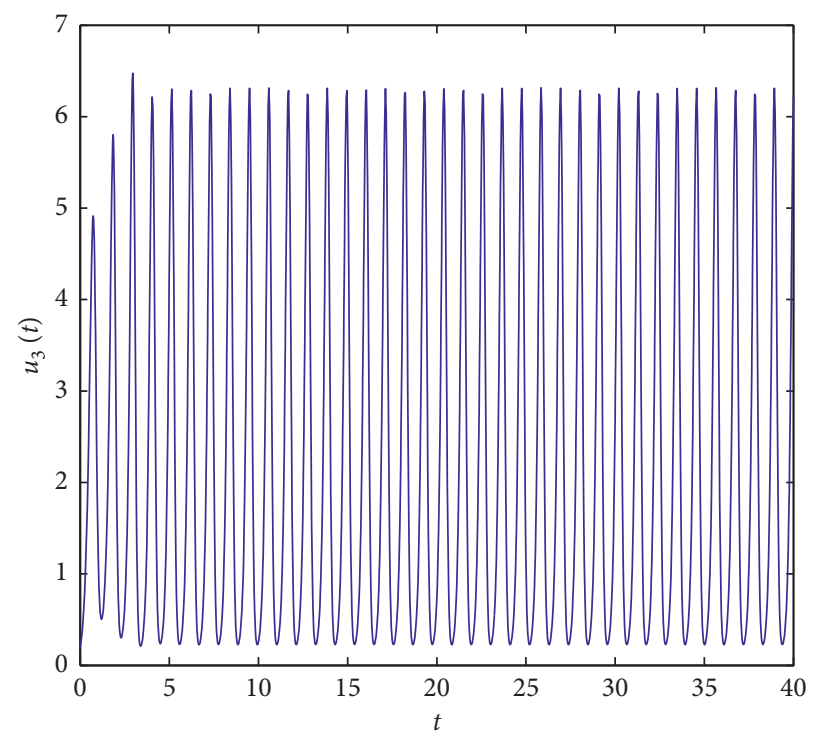

Figure 6: State variable $u_{3}(t)$ versus time $t$ when $\sigma=0.33>\sigma_{0}=0.192$.

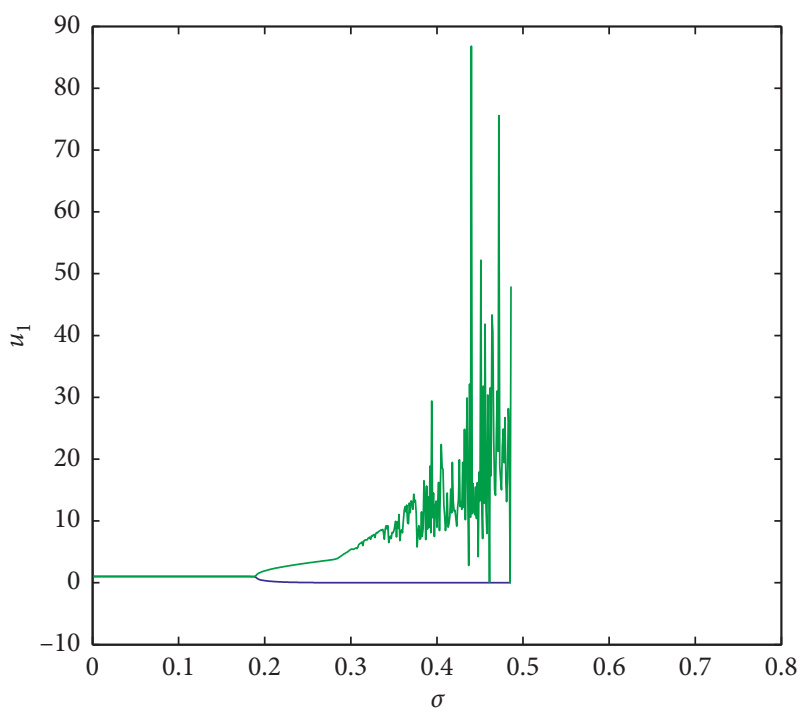

Figure 7: Bifurcation diagram for system (58): $\sigma$ versus $u_{1}$. 


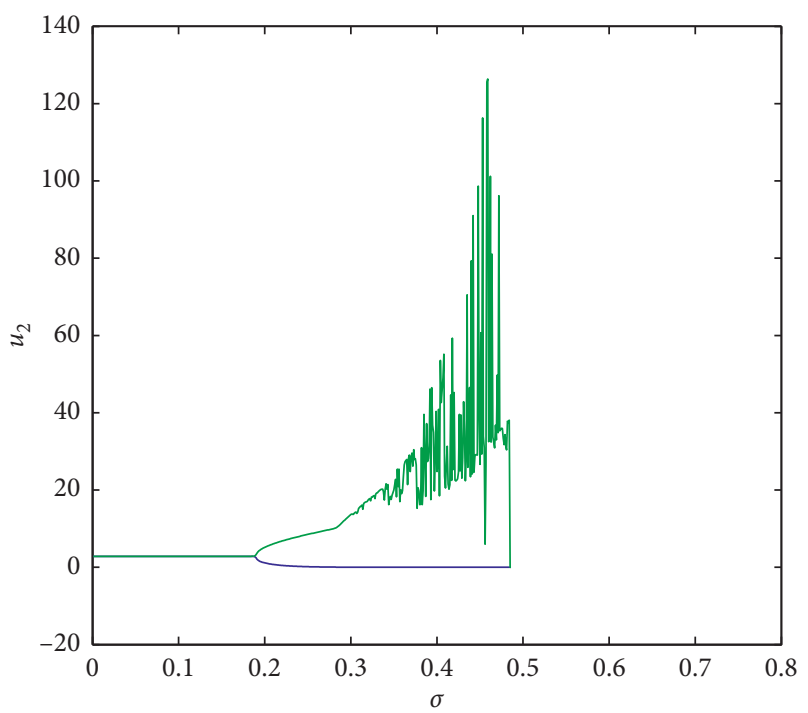

FIgURE 8: Bifurcation diagram for system (58): $\sigma$ versus $u_{2}$.

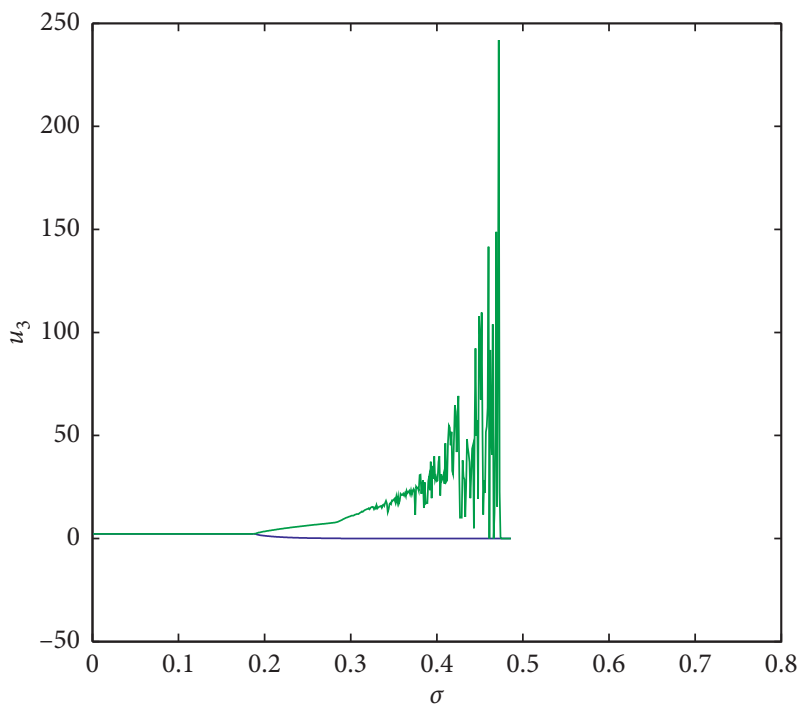

FIgURE 9: Bifurcation diagram for system (58): $\sigma$ versus $u_{3}$.

TABLE 1: The magnitude relation of $\varphi_{0}$ and $\sigma_{0}$ for system (58).

\begin{tabular}{lc}
\hline$\varphi_{0}$ & $\sigma_{0}$ \\
\hline 0.3127 & 0.1920 \\
0.3499 & 0.2173 \\
0.4957 & 0.3218 \\
0.5200 & 0.3400 \\
0.6880 & 0.4732 \\
0.7832 & 0.5543 \\
1.0108 & 0.7651 \\
1.0483 & 0.8022 \\
1.1556 & 0.9123 \\
\hline
\end{tabular}

\section{Conclusions}

Based on the previous works on predator-prey models and noticing the effect of feedback delay between the predators and preys, we have established a new fractional-order competitor-competitor-mutualist Lotka-Volterra model. We have found the conditions to ensure the existence, uniqueness, and nonnegativity of the involved fractionalorder prey-predator model. Applying the Laplace transform, stability theorem, and Hopf bifurcation theory of fractionalorder dynamical systems, we set up a novel sufficient criterion to guarantee the local stability and the emergence of Hopf bifurcation of the involved fractional-order predatorprey system. The study shows that the time delay has a vital effect on controlling the stability. To check the rationality of theoretical predictions, we design the program to implement simulation experiments. The established analytical conclusions have crucial guiding significance in preserving the coexistence of biological populations. In this paper, we assume that the feedback time delay of different species is same. Of course, we can also deal with the different delay cases. We will focus on this topic in near future. 


\section{Data Availability}

No data were used to support this study.

\section{Conflicts of Interest}

The author declares that they have no competing interest.

\section{References}

[1] M. Das, A. Maiti, and G. P. Samanta, "Stability analysis of a prey-predator fractional order model incorporating prey refuge," Ecological Genetics and Genomics, vol. 7-8, pp. 33-46, 2018.

[2] Z. Wang, Y. Xie, J. Lu, and Y. Li, "Stability and bifurcation of a delayed generalized fractional-order prey-predator model with interspecific competition," Applied Mathematics and Computation, vol. 347, pp. 360-369, 2019.

[3] J. Alidousti and E. Ghafari, "Dynamic behavior of a fractional order prey-predator model with group defense, Chaos," Solitons and Fractals, vol. 134, Article ID 109688, 2020.

[4] S. K. Sasmal and Y. Takeuchi, "Dynamics of a predator-prey system with fear and group defense," Journal of Mathematical Analysis and Applications, vol. 481, no. 11, Article ID 123471, 2020.

[5] K. Ryu and W. Ko, "Asymptotic behavior of positive solutions to a predator-prey elliptic system with strong hunting cooperation in predators," Physica A: Statistical Mechanics and Its Applications, vol. 531, Article ID 121726, 2019.

[6] J. S. Guo, K. I. Nakamura, T. Ogiwara, and C. C. Wu, "Traveling wave solutions for a predator-prey system with two predators and one prey," Nonlinear Analysis: Real World Applications, vol. 54, Article ID 103111, 2020.

[7] H. Zhang, Y. Cai, S. Fu, and W. Wang, "Impact of the fear effect in a prey-predator model incorporating a prey refuge," Applied Mathematics and Computation, vol. 356, pp. 328-337, 2019.

[8] T. F. Weng, H. J. Yang, C. G. Gu, J. Zhang, and M. Small, "Predator-prey games on complex networks," Communications in Nonlinear Science and Numerical Simulation, vol. 79, Article ID 104911, 2019.

[9] A. M. C. Sauve, R. A. Taylor, and F. Barraquand, "The effect of seasonal strength and abruptness on predatorCprey dynamics," Journal of Theoretical Biology, vol. 491, Article ID 110175, 2020.

[10] V. Tiwari, J. P. Tripathi, S. Mishra, and R. K. Upadhyay, "Modeling the fear effect and stability of non-equilibrium patterns in mutually interfering predator-prey systems," Applied Mathematics and Computation, vol. 371, Article ID 124948, 2020.

[11] D. Pal, T. K. Kar, A. Yamauchi, and B. Ghosh, "Balancing maximum sustainable yield and ecological resilience in an exploited two-predator one-prey system," Biosystems, vol. 187, Article ID 104064, 2020.

[12] J. Li, X. Zhu, X. Lin, and J. Li, "Impact of cannibalism on dynamics of a structured predator-prey system," Applied Mathematical Modelling, vol. 78, pp. 1-19, 2020.

[13] M. Chen, R. Wu, B. Liu, and L. Chen, "Spatiotemporal dynamics in a ratio-dependent predator-prey model with time delay near the Turing-Hopf bifurcation point," Communications in Nonlinear Science and Numerical Simulation, vol. 77, pp. 141-167, 2019.

[14] P. Yang, "Hopf bifurcation of an age-structured prey-predator model with Holling type II functional response incorporating a prey refuge," Nonlinear Analysis: Real World Applications, vol. 49, pp. 368-385, 2019.

[15] F. Y. Wei and Q. Y. Fu, "Hopf bifurcation and stability for predator-prey systems with Beddington-DeAngelis type functional response and stage structure for prey incorporating refuge," Applied Mathematical Modelling, vol. 40, no. 11, pp. 126-134, 2016.

[16] Y. Song and S. Yuan, "Bifurcation analysis in a predator-prey system with time delay," Nonlinear Analysis: Real World Applications, vol. 7, no. 2, pp. 265-284, 2006.

[17] Y. Song and X. Zou, "Spatiotemporal dynamics in a diffusive ratio-dependent predator-prey model near a Hopf-Turing bifurcation point," Computers and Mathematics with Applications, vol. 67, no. 10, pp. 1978-1997, 2014.

[18] Y. Song, S. Wu, and H. Wang, "Spatiotemporal dynamics in the single population model with memory-based diffusion and nonlocal effect," Journal of Differential Equations, vol. 267, no. 11, pp. 6316-6351, 2019.

[19] D. Duan, B. Niu, and J. Wei, "Hopf-Hopf bifurcation and chaotic attractors in a delayed diffusive predator-prey model with fear effect," Chaos, Solitons and Fractals, vol. 123, pp. 206-216, 2019.

[20] J. Wang, J. Wei, and J. Shi, "Global bifurcation analysis and pattern formation in homogeneous diffusive predator-prey systems," Journal of Differential Equations, vol. 260, no. 4, pp. 3495-3523, 2016.

[21] S. Guo, "Bifurcation and spatio-temporal patterns in a diffusive predator-prey system," Nonlinear Analysis: Real World Applications, vol. 42, pp. 448-477, 2018.

[22] S. J. Guo and S. L. Yan, "Hopf bifurcation in a diffusive LotkaVolterra type system with nonlocal delay effect," Journal of Differential Equations, vol. 260, no. 15, pp. 781-817, 2016.

[23] M. Das and G. P. Samanta, "A delayed fractional order food chain model with fear effect and prey refuge," Mathematics and Computers in Simulation, vol. 178, pp. 218-245, 2020.

[24] M. Das and G. P. Samanta, "A fractional order COVID-19 epidemic transmission model: stability analysis and optimal control," June 5, 2020. Available at SSRN: https://ssrn.com/ abstract $=36359383$.

[25] M. Das and G. P. Samanta, "A prey-predator fractional order model with fear effect and group defense," International Journal of Dynamics and Control, vol. 9, pp. 334-349, 2020.

[26] C. J. Xu, "Delay-induced oscillations in a competitor-competitor-mutualist Lotka-Volterra model," Complexity, vol. 2017, p. 12, Article ID 2578043, 2017.

[27] X. Lv, P. Yan, and S. Lu, "Existence and global attractivity of positive periodic solutions of competitor-competitor-mutualist Lotka-Volterra systems with deviating arguments," Mathematical and Computer Modelling, vol. 51, no. 5-6, pp. 823-832, 2010.

[28] I. Oztuk and F. Ozkose, "Stability analysis of fractional order mathematical model of tumor-immune system interaction, Chaos," Solitons and Fractals, vol. 133, Article ID 109614, 2020.

[29] W. Zhou, C. Huang, M. Xiao, and J. Cao, "Hybrid tactics for bifurcation control in a fractional-order delayed predatorprey model," Physica A: Statistical Mechanics and Its Applications, vol. 515, pp. 183-191, 2019.

[30] C. Huang, H. Li, and J. Cao, "A novel strategy of bifurcation control for a delayed fractional predator-prey model," Applied Mathematics and Computation, vol. 347, pp. 808-838, 2019.

[31] M. Javidi and N. Nyamoradi, "Dynamic analysis of a fractional order prey-predator interaction with harvesting," $A p$ plied Mathematical Modelling, vol. 37, no. 20-21, pp. 8946-8956, 2013. 
[32] S. Mondal, M. Biswas, and N. Bairagi, "Local and global dynamics of a fractional-order predator-prey system with habitat complexity and the corresponding discretized fractional-order system," Journal of Applied Mathematics and Computing, in press, 2020.

[33] H. A. A. El-Saka, S. Lee, and B. Jang, "Dynamic analysis of fractional-order predator-prey biological economic system with Holling type II functional response," Nonlinear Dynamics, vol. 96, no. 1, pp. 407-416, 2019.

[34] H. L. Li, L. Zhang, C. Hu, Y. L. Jiang, and Z. D. Teng, "Dynamical analysis of a fractional-order predator-prey model incorporating a prey refuge," Journal of Applied Mathematics and Computing, vol. 54, no. 1-2, pp. 435-449, 2017.

[35] A. A. Elsadany and A. E. Matouk, "Dynamical behaviors of fractional-order Lotka-Volterra predator-prey model and its discretization," Journal of Applied Mathematics and Computing, vol. 49, no. 1-2, pp. 269-283, 2015.

[36] F. A. Rihan, S. Lakshmanan, A. H. Hashish, R. Rakkiyappan, and E. Ahmed, "Fractional-order delayed predator-prey systems with Holling type-II functional response," Nonlinear Dynamics, vol. 80, no. 1-2, pp. 777-789, 2015.

[37] Y. K. Xie, Z. Wang, B. Meng, and X. Huang, "Dynamical analysis for a fractional-order prey-predator model with Holling III type functional response and discontinuous harvest," Applied Mathematics Letters, vol. 106, Article ID 106342, 2020.

[38] J. Alidousti, "Stability and bifurcation analysis for a fractional prey-predator scavenger model," Applied Mathematical Modelling, vol. 81, pp. 342-355, 2020.

[39] C. Xu, M. Liao, P. Li, Y. Guo, Q. Xiao, and S. Yuan, "Influence of multiple time delays on bifurcation of fractional-order neural networks," Applied Mathematics and Computation, vol. 361, pp. 565-582, 2019.

[40] M. Xiao, W. X. Zheng, J. Lin, G. Jiang, L. Zhao, and J. Cao, "Fractional-order PD control at Hopf bifurcations in delayed fractional-order small-world networks," Journal of the Franklin Institute, vol. 354, no. 17, pp. 7643-7667, 2017.

[41] A. K. O. Tiba and A. F. R. Araujo, "Control strategies for Hopf bifurcation in a chaotic associative memory," Neurocomputing, vol. 323, pp. 157-174, 2019.

[42] B. Tao, M. Xiao, Q. Sun, and J. Cao, "Hopf bifurcation analysis of a delayed fractional-order genetic regulatory network model," Neurocomputing, vol. 275, pp. 677-686, 2018.

[43] H. Li, C. D. Huang, and T. X. Li, "Dynamic complexity of a fractional-order predator-prey system with double delays," Physica A: Statistical Mechanics and Its Applications, vol. 526, Article ID 120852, 2019.

[44] F. A. Rihan and G. Velmurugan, "Dynamics of fractionalorder delay differential model for tumor-immune system, Chaos," Solitons \& Fractals, vol. 132, Article ID 109592, 2020.

[45] I. Podlubny, Fractional Differential Equations, Academic Press, New York, NY, USA, 1999.

[46] B. Bandyopadhyay and S. Kamal, Stablization and Control of Fractional Order Systems: A Sliding Mode Approach, Vol. 317, Springer, Heidelberg, Germany, 2015.

[47] Y. Li, Y. Q. Chen, and I. Podlubny, "Stability of fractionalorder nonlinear dynamic systems: Lyapunov direct method and generalized Mittag-Leffler stability," Computers and Mathematics with Applications, vol. 59, no. 5, pp. 1810-1821, 2009.

[48] Z. M. Odibat and N. T. Shawagfeh, "Generalized Taylor's formula," Applied Mathematics and Computation, vol. 186, no. 1, pp. 286-293, 2007.
[49] D. Matignon, "Stability results for fractional differential equations with applications to control processing," Computational Engineering in Systems Applications, vol. 2, pp. 963968, 1996.

[50] X. Wang, Z. Wang, and J. Xia, "Stability and bifurcation control of a delayed fractional-order eco-epidemiological model with incommensurate orders," Journal of the Franklin Institute, vol. 356, no. 15, pp. 8278-8295, 2019.

[51] W. Deng, C. Li, and J. Lü, "Stability analysis of linear fractional differential system with multiple time delays," Nonlinear Dynamics, vol. 48, no. 4, pp. 409-416, 2007.

[52] F. A. Rihan, S. Lakshmanan, A. H. Hashish, R. Rakkiyappan, and E. Ahmed, "Fractional-order delayed predator-prey systems with Holling type-II functional response," Nonlinear Dynamics, vol. 80, no. 1-2, pp. 777-789, 2015. 